\title{
Nanoemulsion of high DHA vegetarian algal oil enhances DHA bioavailability - a randomised crossover trial
}

\author{
K. E. Lane ${ }^{1}$, W. Li ${ }^{2}$, C. Smith ${ }^{2}$ and E. J. Derbyshire ${ }^{3}$ \\ ${ }^{1}$ Faculty of Education, Health \& Community, Centre for Tourism, Events \& Food Studies, Liverpool John Moores \\ University, Liverpool, United Kingdom L17 6BD, ${ }^{2}$ Department of Food and Tourism Management, Manchester \\ Metropolitan University (MMU), Manchester, M15 6BG and ${ }^{3}$ School of Healthcare Science,
} $M M U$, Manchester M1 5DG

Research has identified that vegetarians/vegans, non-fish eaters and pregnant women may not consume adequate quantities of long chain omega-3 polyunsaturated fatty acids (LC3PUFA) ${ }^{(1-3)}$. Food-based oil in water nanoemulsion systems may offer a potential vehicle to improve LC3PUFA intakes when used to create enriched food products, especially for populations who do not consume oily fish or have restricted intakes. The use of nanoemulsions has been found to improve the bioavailability of lipophilic compounds such as curcumin and delta tocopherol ${ }^{(4,5)}$ but its application to vegetarian sources of LC3PUFAhas not been studied widely.

In a randomised crossover study, eleven volunteers (mean age $33 \cdot 6$ years SD10.3; BMI 23.8SD2.1) were recruited and asked to ingest a: 1) Yogurt drink containing algae oil and water nanoemulsion, providing1264 mg docosahexaenoic acid (DHA), or 2) Formulated yogurt drink containing bulk algae oil providing the same amount of DHA (control).

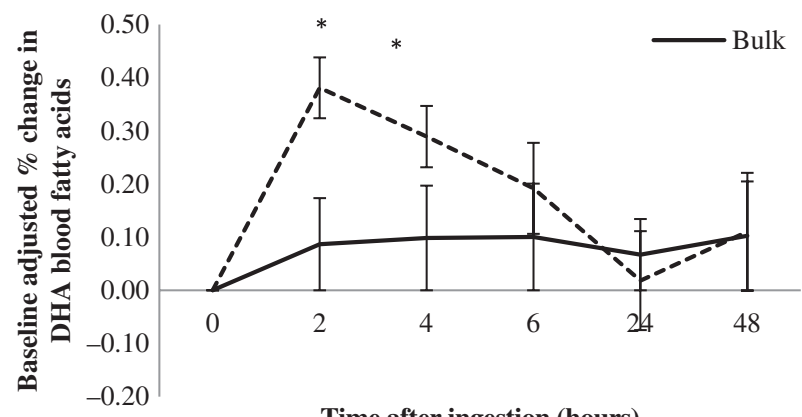

Time after ingestion (hours)

Fatty acid analysis was completed by fingertip blood sampling at 2, 4, 6, 24 and 48 hours with the validated 'Omega Blood Count ${ }^{\mathrm{TM}}$ ' test $\mathrm{kit}^{(6)}$. Percentage blood fatty acid increases were based on incremental area under the curve (iAUC) of baseline adjusted values. Key: Mean baseline adjusted DHAvalues. Error bars represent the standard error (SE).Asterisks denote statistical significance (paired t-test $P=0 \cdot 001$ and $P=0 \cdot 0400$ to 2 and 2 to 4 hours respectively).

Results indicate that the absorption of DHA peaked 2 hours after ingestion for the nanoemulsion, with the iAUC being significantly higher for the nanoemulsion compared with the bulk oil 2 and 4 hours following ingestion $(P=0 \cdot 001$ and $P=0 \cdot 04$ respectively). Larger and longer trials are now needed but this preliminary trial indicates that nanoemulsion of high DHA vegetarian algal oil may help to improve DHA status in groups with low intakes.

This research was conducted in adherence to the Helsinki Declaration. Thanks go to John Dobson at DSM UK and Martek Biosciences Corporation, Columbia for the donation of the 35 per cent DHA S algae oil.

1. Davis BC, Kris-Etherton PM. (2003) Achieving optimal essential fatty acid status in vegetarians: current knowledge and practical implications. Am J Clin Nutr 78, 640S-6S.

2. Derbyshire E. (2009) Functional LC $\omega-3$ PUFAs in pregnancy: a short review of the literature. $J$ of Foodserv 20, 224-9.

3. Welch AA, Shakya-Shrestha S, Lentjes XX et al. (2010) Dietary intake and status of $n-3$ polyunsaturated fatty acids in a population of fish-eating and non-fish-eating meat-eaters, vegetarians, and vegans: results from the EPIC-Norfolk cohort. Am J Clin Nutr 92, 1040-51.

4. Kotyla T, Kuo F, Moolchandani V, et al. (2008) Increased bioavailability of a transdermal application of a nano-sized emulsion preparation. Int $J$ Pharm 347, 144-148.

5. Yu H, Huang Q. (2012) Improving the Oral Bioavailability of Curcumin Using Novel Organogel-Based Nanoemulsions. J Agric Food Chem. 60, 5373-9.

6. Gordon Bell J, Mackinlay EE, Dick JR, et al. (2011) Using a fingertip whole blood sample for rapid fatty acid measurement: method validation and correlation with erythrocyte polar lipid compositions in UK subjects. Br J Nutr 106, 1408-15. 